\title{
Correction to: Human papillomavirus genotype distribution in Ethiopia: an updated systematic review
}

Awoke Derbie ${ }^{1,2,3^{*}} \mathbb{0}$, Daniel Mekonnen ${ }^{1,3}$, Endalkachew Nibret ${ }^{3,4}$, Melanie Maier $^{5}$, Yimtubezinash Woldeamanuel ${ }^{2,6}$ and Tamrat Abebe ${ }^{6}$

\section{Correction to: Virology Journal (2022) 19:13} https://doi.org/10.1186/s12985-022-01741-1

The original article has been corrected.

Following publication of the original article [1], the authors identified an error in Fig. 2. The correct figure is given below. 


\section{HPV genotype distribution in Ethiopia, \%}

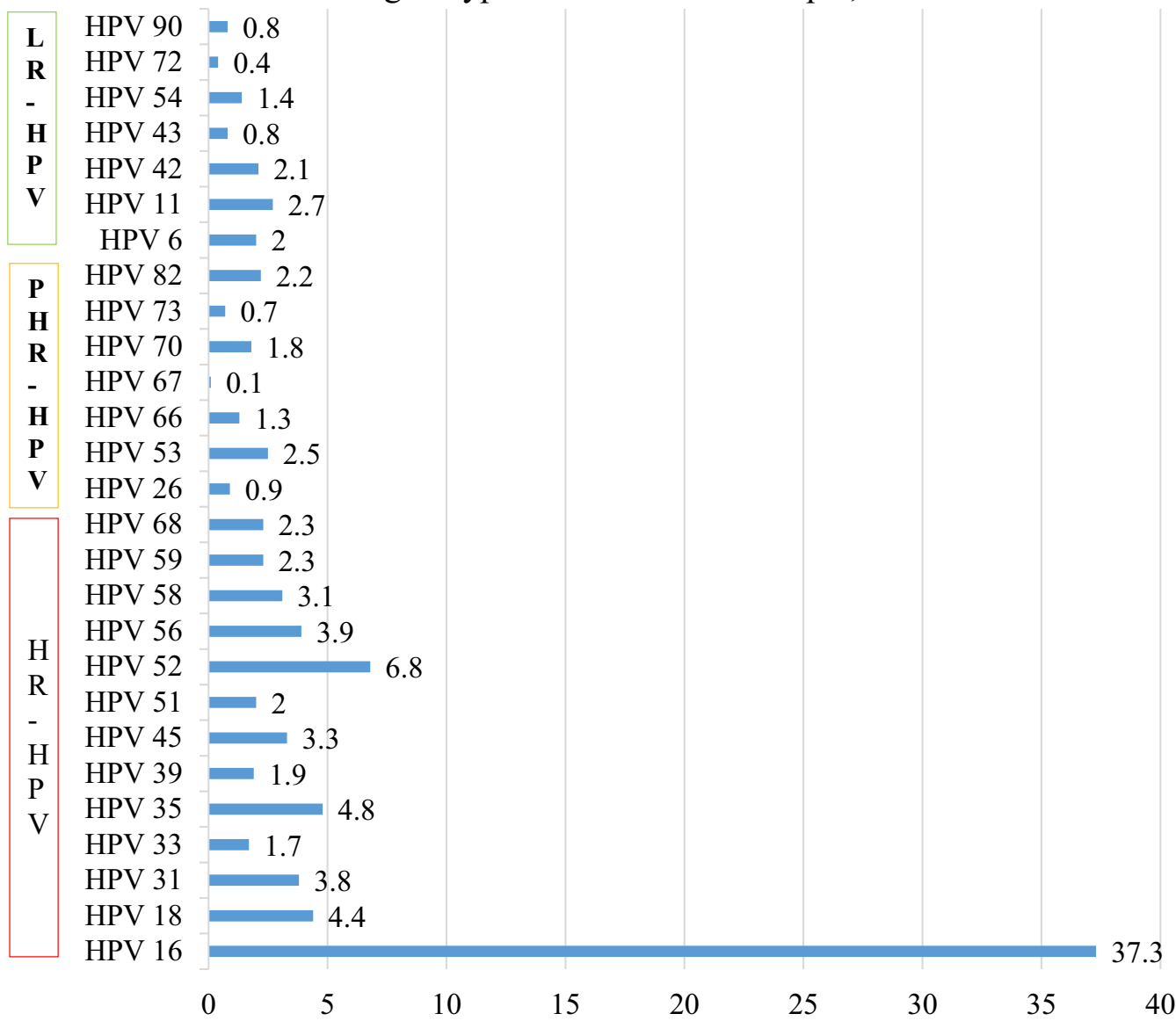

HPV genotype distribution in Ethiopia, \%

Fig. 2 Frequency of HPV genotypes identified from the included studies, 2005-2019. LR low-risk, PHR probable high-risk, HR high-risk, HPV human papillomavirus

\section{Author details}

${ }^{1}$ Department of Medical Microbiology, College of Medicine and Health Sciences, Bahir Dar University, Bahir Dar, Ethiopia. ${ }^{2}$ Centre for Innovative Drug Development and Therapeutic Trials for Africa (CDT-Africa), Addis Ababa University, Addis Ababa, Ethiopia. ${ }^{3}$ Department of Health Biotechnology, Institute of Biotechnology, Bahir Dar University, Bahir Dar, Ethiopia. ${ }^{4}$ College of Science, Bahir Dar University, Bahir Dar, Ethiopia. ${ }^{5}$ Department of Diagnostics, Institute of Virology, Leipzig University Hospital, Leipzig, Germany. ${ }^{6}$ Department of Medical Microbiology, Immunology and Parasitology, College of Health Sciences, Addis Ababa University, Addis Ababa, Ethiopia.

Published online: 07 February 2022

\section{Publisher's Note}

Springer Nature remains neutral with regard to jurisdictional claims in published maps and institutional affiliations.

\section{Reference}

1. Derbie A, Mekonnen D, Nibret E, Maier M, Woldeamanuel Y, Abebe T. Human papillomavirus genotype distribution in Ethiopia: an updated systematic review. Virol J. 2022;19:13. https://doi.org/10.1186/ s12985-022-01741-1. 